\title{
Pathophysiology and surgical treatment of spinal adhesive arachnoid pathology: patient series
}

\author{
Izumi Koyanagi, MD, ${ }^{1}$ Yasuhiro Chiba, MD, ${ }^{1}$ Genki Uemori, MD, ${ }^{2}$ Hiroyuki Imamura, MD, ${ }^{1}$ Masami Yoshino, MD, ${ }^{1}$ and \\ Toshimitsu Aida, $\mathrm{MD}^{1}$
}

\begin{abstract}
${ }^{1}$ Department of Neurosurgery, Hokkaido Neurosurgical Memorial Hospital, Sapporo, Hokkaido, Japan; and ${ }^{2}$ Department of Neurosurgery, Moriyama Hospital, Asahikawa, Hokkaido, Japan
\end{abstract}

\begin{abstract}
BACKGROUND Spinal adhesive arachnoid pathology is a rare cause of myelopathy. Because of rarity and variability, mechanisms of myelopathy are unknown. The authors retrospectively analyzed patients to understand pathophysiology and provide implications for surgical treatment.

OBSERVATIONS Nineteen consecutive patients were studied. Thirteen patients had a secondary pathology due to etiological disorders such as spinal surgery or hemorrhagic events. They received arachnoid lysis (4 patients), syringo-subarachnoid (S-S) shunt (8 patients) with or without lysis, or anterior decompression. Three of them developed motor deterioration after lysis, and 6 patients needed further 8 surgeries. Another 6 patients had idiopathic pathology showing dorsal arachnoid cyst formation at the thoracic level that was surgically resected. With mean follow-up of 44.3 months, only 4 patients with the secondary pathology showed improved neurological grade, whereas all patients with idiopathic pathology showed improvement. LESSONS The idiopathic pathology was the localized dorsal arachnoid adhesion that responded to surgical treatment. The secondary pathology produced disturbed venous circulation of the spinal cord by extensive adhesions. Lysis of the thickened fibrous membrane with preservation of thin arachnoid over the spinal veins may provide safe decompression. S-S shunt was effective if the syrinx extended to the level of normal subarachnoid space.
\end{abstract}

https://thejns.org/doi/abs/10.3171/CASE21426

KEYWORDS spinal adhesive arachnoiditis; syringomyelia; spinal arachnoid cyst; syringo-subarachnoid shunt; surgical treatment

Spinal adhesive arachnoid pathology is a relatively rare cause of myelopathy. It is known that infectious or traumatic disorders cause extensive adhesions of the spinal subarachnoid space. ${ }^{1}$ A more localized form results in cystic dilatation of the dorsal subarachnoid space compressing the spinal cord. ${ }^{2,3}$ The latter pathology has often been reported as spinal arachnoid cyst ${ }^{2,3}$ or arachnoid web. ${ }^{4}$ Syrinx formation is common in any form of spinal adhesive arachnoid pathology. However, because of the rarity and variability of this disorder, the mechanisms of myelopathy or syrinx formation have not been clarified. Surgical treatment for extensive adhesions of the spinal subarachnoid space was difficult, and long-term control of the disease could not be achieved. $^{1,5}$ In this study, we retrospectively analyzed recent surgical series of our patients with spinal adhesive arachnoid pathology, with the purpose being to understand the pathophysiology underlying the arachnoid pathology and provide implications for surgical treatment.

\section{Study Description}

Nineteen consecutive patients who received surgical treatment of spinal adhesive arachnoid pathology from 2010 to 2020 were retrospectively studied. There were 8 men and 11 women aged 24 to 84 years (mean 58.8 , median 59) at time of surgery. Initial symptoms of adhesive arachnoid pathology were thoracic or back pain (6 patients), neck pain (1 patient), upper limb pain and motor weakness (1 patient), paraparesis (7 patients), tetraparesis ( 1 patient), and numbness of legs (3 patients). The interval from onset of symptoms to admission to our hospital ranged from 1 to 162 months (mean 39.1, median 16.0). On admission, 18 of 19 patients presented with paraparesis or tetraparesis of various degrees, and 1 patient showed severe thoracic pain. In all patients, magnetic resonance imaging (MRI) was used for diagnosis of arachnoid pathology.

Etiology of arachnoid adhesion, neurological state by McCormick (MC) grade (grade l: neurologically normal or mild deficits, normal

ABBREVIATIONS CSF = cerebrospinal fluid; $M C=$ McCormick; MRI = magnetic resonance imaging; $S-S=$ syringo-subarachnoid INCLUDE WHEN CITING Published October 18, 2021; DOI: 10.3171/CASE21426.

SUBMITTED July 23, 2021. ACCEPTED August 13, 2021.

(c) 2021 The authors, CC BY-NC-ND 4.0 (http://creativecommons.org/licenses/by-nc-nd/4.0/). 
gait; grade II: sensorimotor deficits, independent ambulation; grade III: more severe deficits requiring cane/brace for ambulation; grade IV: severe deficits requiring wheelchair), ${ }^{6}$ MRI findings, surgical treatment, and follow-up results were analyzed.

\section{Etiology of Spinal Arachnoid Adhesion}

Etiologically, 13 of 19 patients had a medical history that resulted in arachnoid adhesions and so they were regarded as the secondary pathology. Spinal surgery was most common (repair of ventral dural defect showing idiopathic spinal cord herniation using autologous fascia or artificial dura in 2 patients; surgeries for cervical spondylosis in 2 patients). The next most common etiology was intradural hemorrhagic disorder (3 patients). Two of the 3 patients had intracranial subarachnoid hemorrhage from ruptured basilar aneurysm or dissection of the vertebral artery. The other patient suffered from acute spinal subdural hematoma of unknown origin. Trauma as the etiology was noted in 2 patients (severe head injury in 1; spinal cord injury in 1). Another 4 patients had different types of etiology: tuberculosis (caries), pyogenic meningitis, oilmyelography, and cerebrospinal fluid (CSF) leakage syndrome. The interval from etiological events to onset of symptoms of arachnoid adhesion in these 13 patients ranged from 1 to 744 months (mean 167.3 months, median 84 months). Onset of symptoms to admission ranged from 1 to 161 months (mean 38.6 months, median 14 months).

Six of 19 patients did not have etiological disorders and were regarded as having idiopathic pathology. They gradually presented with thoracic or back pain (6 patients) and/or mild paraparesis (5 patients). Onset of symptoms to admission ranged from 4 to 120 months (mean 40.2 months, median 26.0 months).

\section{Neurological State on Admission}

The neurological state of 19 patients on admission was MC grade IV (6 patients), grade III (5 patients), and grade II (8 patients) (Table 1). Thirteen patients with secondary pathology generally presented with more severe neurological deficits (MC grade IV, 6 patients; grade III, 4 patients; and grade II, 3 patients) than 6 patients with idiopathic pathology (MC grade III, 1 patient; grade II, 5 patients). Severe deficits (MC grades III and IV) were significantly more common in patients with secondary pathology (10 of 13 patients, 83.3\%) than idiopathic pathology (1 of 6 patients, $16.7 \% ; p=0.0408$ by Fisher's exact test).

\section{MRI Findings}

The spinal levels of arachnoid adhesion in 19 patients estimated from MRI findings were cervical in 1 patient, cervical to thoracic in 3 patients, cervical to lumbar in 1 patient, thoracic in 13 patients, and thoracic to lumbosacral in 1 patient (Table 1). Extension of adhesion ranged from 2 to 16 spinal levels (mean 5.7 levels). Thirteen patients with secondary pathology had significantly longer levels of adhesion ( 3 to 16 levels; mean 7.2 levels) compared to 6 patients with idiopathic pathology (2 to 3 levels; mean 2.5 levels) by unpaired Student's $t$-test $(p=0.0195)$.

All 13 patients with the secondary pathology showed intramedullary hyperintensity on T2-weighted images, indicating increased interstitial fluid. Eleven of them (84.6\%) showed syrinx formation (Figs. 1 and 2). The spinal cord was swollen in appearance with the irregularly narrowed subarachnoid space at the affected levels. Local dilatation of the subarachnoid space (arachnoid cyst formation) was also present in 8 of 13 patients (ventral, 6 patients; dorsolateral, 1 patient; ventral and dorsal, 1 patient).

TABLE 1. Summary of 19 patients with spinal adhesive arachnoid pathology

\begin{tabular}{cccccccccc}
\hline Case No. & Etiology & MC & Adhesion Levels & IMH & Syrinx & Cyst & Surgery & F/U & Final MC \\
\hline 1 & SHI & IV & T3-11 & T3-12 & T4-11 & T3-4 ventral & Lysis, lysis & 49 & IV \\
\hline 2 & SAH & IV & T5-12 & T5-12 & T5-12 & - & Lysis, S-S & 29 & IV \\
\hline 3 & Tb & IV & T3-12 & T4-11 & T4-11 & - & Lysis, S-S & 5 & IV \\
\hline 4 & MLG & III & T8-10 & T10-11 & T10 & T8-9 dorsolateral & Lysis & 72 & II \\
\hline 5 & Surgery & III & T2-5 & C6-T9 & C6-T4 & T5 ventral & Lysis, S-S & 89 & III \\
\hline 6 & SCI & IV & C4-6 & C1-6 & C1-6 & - & Lysis, S-S & 82 & IV \\
\hline 7 & Surgery & IV & C4-T1 & C1-T4 & C2-5 & C5 ventral & S-S, LN & 67 & IV \\
\hline 8 & SAH & IV & T6-11 & T7-9 & - & T6-7 ventral & Lysis & 50 & III \\
\hline 9 & Surgery & II & C7-T2 & C7-T5 & C7-T5 & - & Lysis, S-S & 69 & II \\
\hline 10 & SDH & III & T4-S1 & T5-12 & - & T4-5 ventral, T6-9 dorsal & Lysis & 56 & IV \\
\hline 11 & Surgery & III & C4-T1 & C4-T4 & T3-4 & C3-4 ventral & AF, S-S, lysis & 12 & III \\
\hline 12 & CSFL & II & C5-L1 & C6-L1 & T2-12 & C5-T2 ventral & Lysis, S-S, lysis & 6 & II \\
\hline 13 & Meningitis & II & T2-7 & C5-T8 & C5-T8 & T4-5 ventral & Lysis, S-S & 6 & I \\
\hline 14 & - & III & T3-5 & C4-T4 & T1-4 & T4-5 dorsal & Cyst removal & 3 & II \\
\hline 15 & - & II & T5-7 & - & - & T5-7 dorsal & Cyst removal & 77 & I \\
\hline 16 & - & II & T2-3 & T1-3 & T1-3 & T2-3 dorsal & Cyst removal & 24 & I \\
\hline 17 & - & II & T5-6 & - & - & T5-6 dorsal & Cyst removal & 59 & I \\
\hline 18 & - & II & T2-3 & T2-3 & - & T2-3 dorsal & Cyst removal & 30 & I \\
\hline 19 & - & II & T2-4 & - & - & T2-4 dorsal & Cyst removal & 17 & I \\
\hline
\end{tabular}

$\mathrm{AF}=$ anterior fusion; $\mathrm{CSFL}=$ cerebrospinal fluid leakage syndrome; $\mathrm{Cyst}=$ cyst formation of subarachnoid space; Final $\mathrm{MC}=\mathrm{McCormick}$ grade at the final follow-up from the last surgery; F/U = follow-up period (months) from the last surgery; IMH = intramedullary hyperintensity; $\mathrm{LN}=$ laminectomy; $\mathrm{MC}=\mathrm{McCormick}$ grade; $\mathrm{SAH}=$ subarachnoid hemorrhage; $\mathrm{SDH}=$ subdural hematoma; $\mathrm{SHI}=$ severe head injury.

Cases 1 to 13 had past history of etiological disorders (secondary arachnoid pathology). Cases 14 to 19 had no etiological disorders (idiopathic arachnoid pathology). 

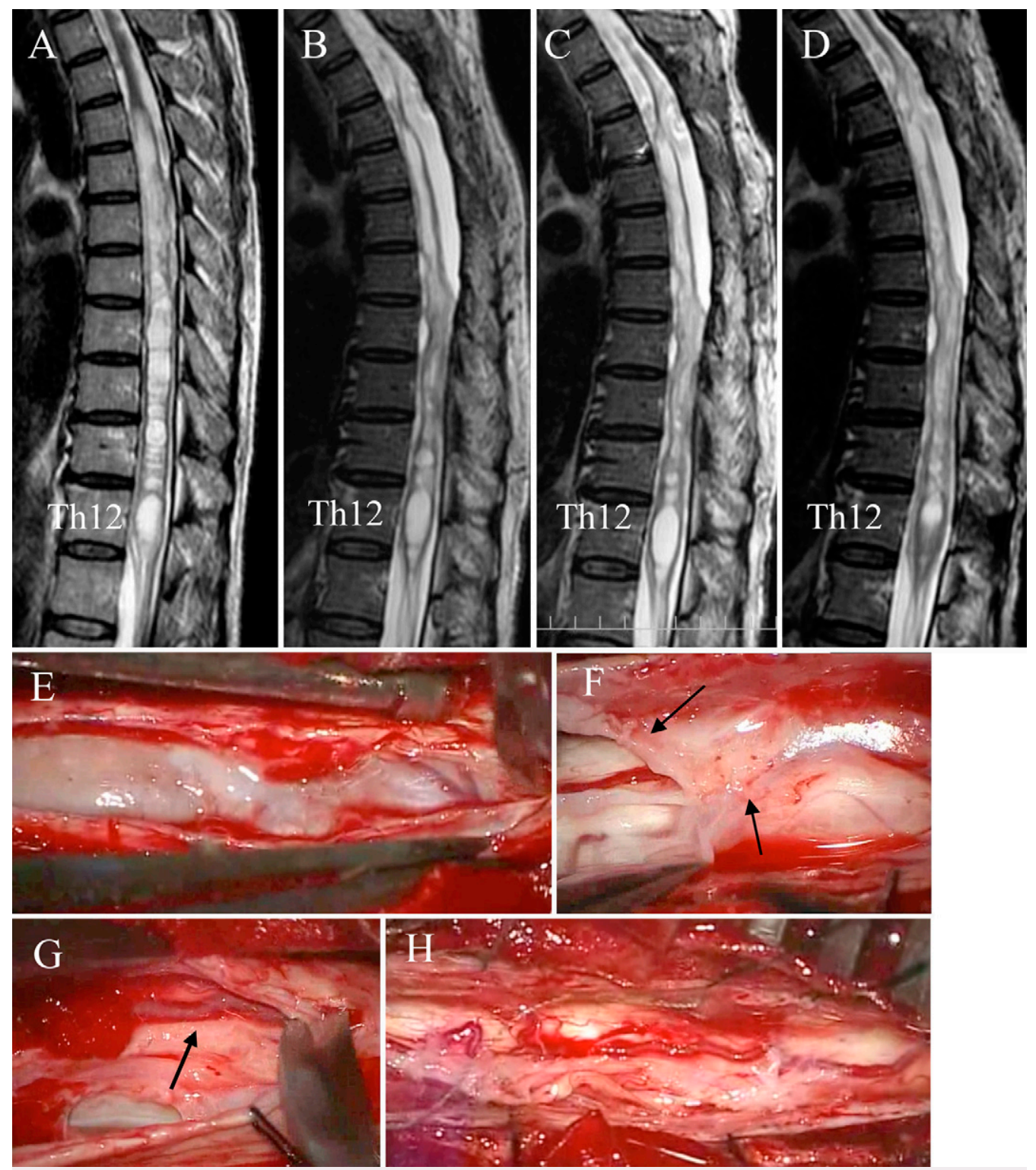

FIG. 1. Pre- and postoperative MRI (T2-weighted sagittal image of the thoracic spine) (A-D) and intraoperative photographs (E-G) of the first surgery of Case 2. This female patient presented with paraparesis that appeared 1 year before admission. She had past history of subarachnoid hemorrhage due to a ruptured basilar aneurysm 6 years earlier. A: Preoperative MRI showed expanding appearance of the spinal cord with intramedullary hyperintensity and syrinx formation at T5-12 levels. B: MRI 78 months after T4-8 lysis of subarachnoid adhesion, with the lysis surgery showing decreased size of the syrinx. She showed improved symptoms after surgery. C: MRI 95 months after surgery showed expansion of the syrinx. She experienced severe back pain and deteriorated paraparesis. D: MRI 26 months after the second surgery (S-S shunt at T11-12) showed decreased size of the syrinx. Back pain improved and paraparesis was slightly recovered. E: Thick arachnoid membrane after opening the dura. F: The thick membrane with rich vascularity (arrows) is compressing the dorsal spinal veins. G: The membrane was carefully dissected to decompress the dorsal spinal vein (arrow). H: After removal of the thick arachnoid membrane. The dorsal spinal cord veins were decompressed. $T h=$ thoracic.

In 6 patients with idiopathic pathology, local dilatation of the dorsal subarachnoid space at the thoracic levels and deformity of the spinal cord were the main findings (Figs. 3 and 4). Intramedullary hyperintensity on T2-weighted image was present in 3 patients, 2 of whom showed syrinx formation (Fig. 3). Thus, the intramedullary hyperintensity and syrinx formation were more common in the patients with secondary pathology (13 and 11 of 13 patients, respectively) than those with idiopathic pathology ( 3 of 6 patients; $p=0.026$; and 2 of 6 patients; $p=0.046$ by Fisher's exact test).

\section{Surgical Treatment}

Of 13 patients with the secondary pathology, 7 patients (Cases $1,2,3,4,8,10$, and 12; Table 1) received laminectomy and lysis of arachnoid adhesion with duraplasty using autologous femoral 

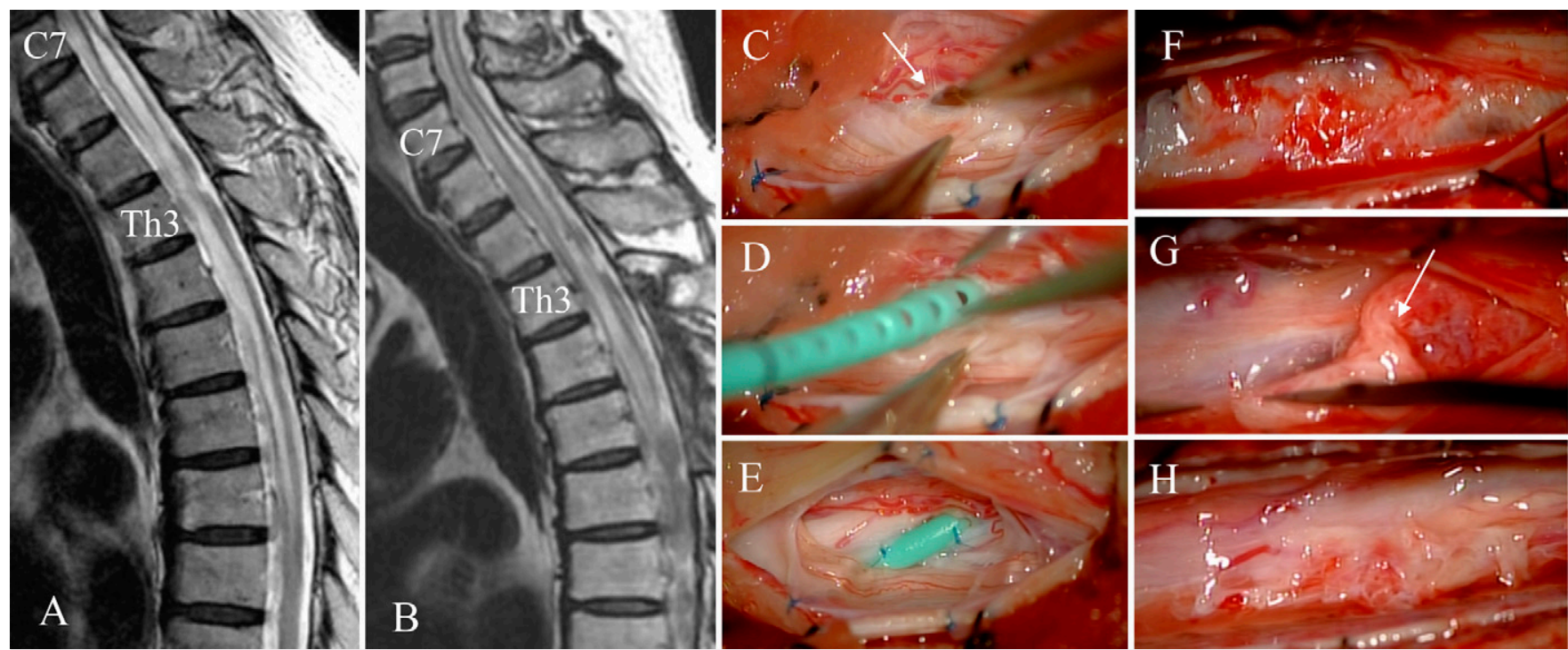

FIG. 2. Pre- and postoperative MRI findings (T2-weighted sagittal images of the cervicothoracic spine (A and B) and intraoperative photographs (C-H) of Case 13. This male patient had a history of pyogenic meningitis 35 years earlier and developed mild paraparesis with left upper limb numbness 13 years before admission. A: Preoperative MRI showing syrinx formation from the cervical to thoracic levels and irregular subarachnoid space at the thoracic levels. This patient received S-S shunt at T1-2 and lysis at T3-5. B: Postoperative MRI 6 months after surgery showing decreased size of the syrinx. The patient showed improved pain and paraparesis after surgery. C: After opening the dura at T1-2, small myelotomy was made at the left dorsal root entry zone (arrow). D: A 1.5-mm S-S shunt tube was introduced into the syrinx. E: The subarachnoid end of the S-S shunt tube was placed in the ventral subarachnoid space. The tube was sutured to the dentate ligament and pia mater using 8-0 nylon. F: On opening the dura at T3-5, hypertrophied arachnoid with thick fibrous component containing rich vascularity was present. G: The fibrous membrane (arrow) was dissected from the arachnoid of the spinal cord surface. H: After removal of thick fibrous membrane, the dorsal arachnoid membrane was preserved with intact subarachnoid vessels.

fascia (Fig. 1). Immediately after surgery, 2 of the patients (Cases 2 and 4) showed improved paraparesis, 3 (Cases 1, 3, and 8) remained unchanged, and 2 (Cases 10 and 12) showed deterioration of paraparesis or unilateral upper limb paresis. During followup, 1 patient (Case 1) received additional laminectomy and arachnoid lysis because of development of thoracic pain 4 months after the first surgery. Three patients (Cases 2, 3, and 12) received syringo-subarachnoid (S-S) shunt 4 to 111 months after surgery because of development of pain/numbness (Cases 2 and 3 ) or progression of paraparesis (Case 12). One of those patients (Case 12) further received lysis surgery because of reexpansion of syrinx with worsening of paraparesis 9 months after the second surgery.

Four patients (Cases 5, 6, 9, and 13) with secondary pathology received lysis of arachnoid adhesion and simultaneous S-S shunt at the level at which there was no subarachnoid adhesion (Fig. 2). All 4 patients showed improved sensory symptoms (numbness or pain) after surgery. Paraparesis was also improved in 1 patient (Case 13), but another patient (Case 9) developed worsened motor paresis of unilateral leg after surgery, presumably due to the subarachnoid lysis procedure.

Another 2 patients with the secondary pathology received S-S shunt (Case 7) or cervical anterior decompression and fusion using autologous iliac bone graft because of remaining bony spur at the previously operated level (Case 11). They showed some improvement in sensory symptoms (neck pain or upper limb numbness) after surgery. However, 1 of them (Case 7) received additional laminectomy 3 months after S-S shunt because of residual neck pain. One other patient (Case 11) received S-S shunt 13 months after the first surgery because of progression of tetraparesis. This patient received further operation with intradural lysis 9 months after S-S shunt as the third surgery.

All 6 patients with idiopathic pathology (Cases 14 to 19; Table 1) received removal of the dorsal subarachnoid cyst wall via laminectomy (Fig. 3) and primary dural closure. They showed improved symptoms, including reduction in back pain (6 patients) and paraparesis (5 patients) after surgery (Figs. 3 and 4 ).

\section{Follow-Up Results}

Follow-up periods of 13 patients with secondary pathology from the last surgery ranged from 5 to 82 months (mean 44.3 months). Compared to the initial preoperative neurological state, only 4 patients (30.8\%) showed improved MC grade (IV to III: 1 patient; III to II: 2 patients; II to I: 1 patient). In 8 patients $(61.5 \%), M C$ grades remained the same (IV to IV: 5 patients; III to III: 2 patients; II to II: 1 patient). One patient $(7.7 \%)$ showed deteriorated MC grade (III to IV) (Table 1). The last MRI (1 to 89 months after the last surgery, mean 34.2 months) revealed that 9 of 11 patients with syringomyelia showed decreased size of syrinx. Two patients without syrinx formation showed slight extension of intramedullary edema.

Follow-up periods of 6 patients with idiopathic pathology from surgery ranged from 3 to 77 months (mean 35.0 months). All 6 patients showed improved MC grade (III to II: 1 patient; II to I: 5 patients) (Table 1). The last MRI (2 to 63 months after surgery, mean 30.8 months) revealed that the dorsal subarachnoid space was normalized in all 6 patients (Figs. 3 and 4). Syrinx (2 patients) and intramedullary edema (3 patients) were also improved (Fig. 3). 


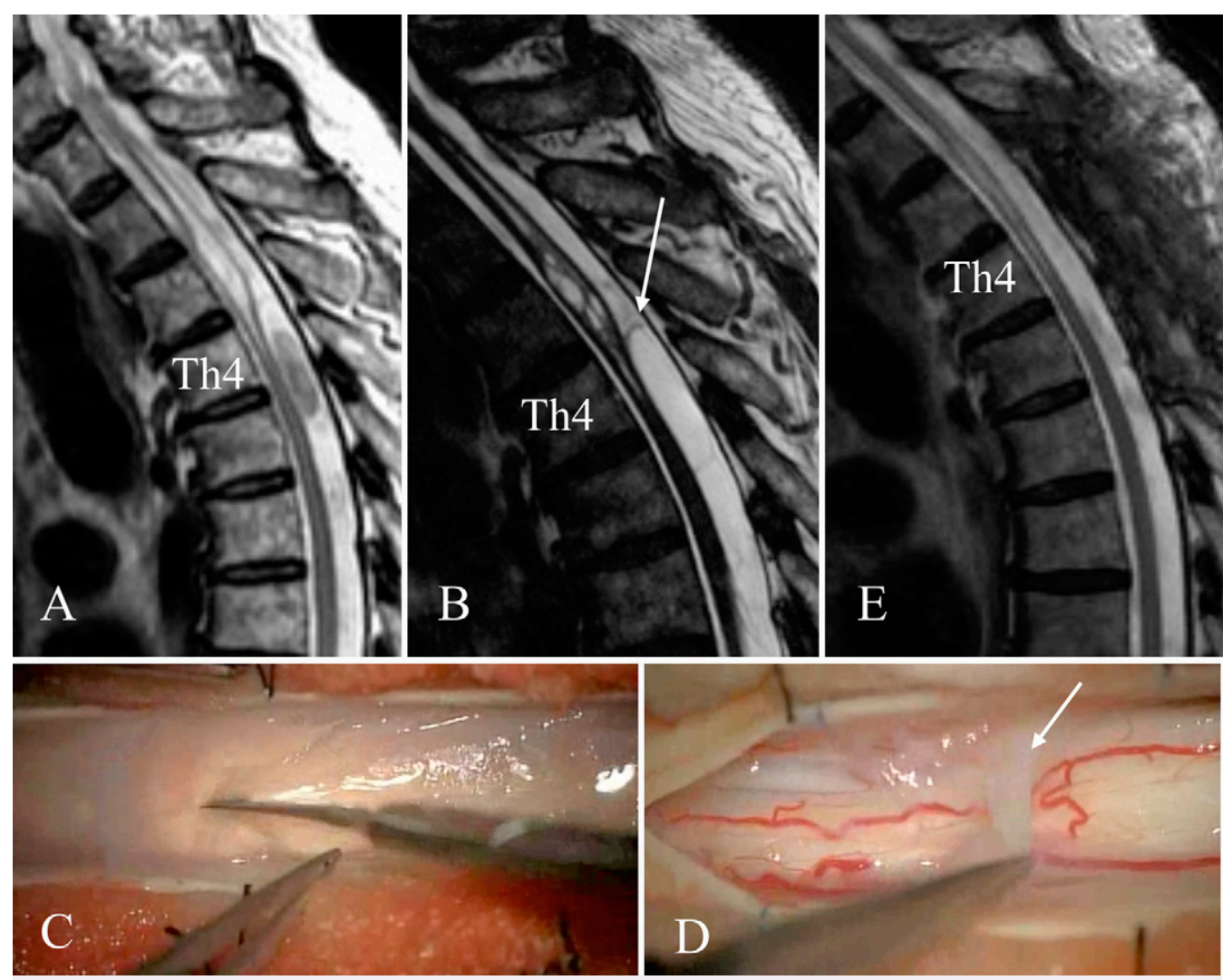

FIG. 3. Pre- and postoperative MRI findings of the thoracic spine and intraoperative photographs of Case 14. This patient presented with paraparesis and severe thoracic pain for 10 years before admission without etiological disorders. A: Preoperative MRI (T2-weighted sagittal image) showed local dilatation of the dorsal subarachnoid space at T4 with rostral syrinx formation. B: Heavy T2-weighted sagittal image (FIESTA) demonstrating cyst wall in the dorsal subarachnoid space (arrow). The patient received T3-5 laminectomy and removal of the subarachnoid cyst wall. C: Intraoperative photograph showing incision of the dorsal arachnoid membrane. D: Thick arachnoid cyst wall (arrow) was present in the subarachnoid space. E: Postoperative MRI (T2-weighted sagittal image) 2 months after surgery showed normalized subarachnoid space with decreased syrinx. The patient had improved pain levels and paraparesis after surgery.

\section{Discussion \\ Observations}

The present study revealed two types of spinal adhesive arachnoid pathology. Extensive subarachnoid adhesion occurred secondary to various disorders affecting the spinal subarachnoid space. Arachnoid membrane was thickened and CSF pathway around the spinal cord was severely occluded. The other type involved relatively local adhesive change at the dorsal subarachnoid space without preceding etiological disorders. Cystic dilatation of the dorsal subarachnoid space with compression of the spinal cord was the main MRI finding. These two types of spinal arachnoid pathology were also reported by Klekamp. ${ }^{5}$ Response to surgical treatment was usually poor for patients with secondary pathology because of extensive spinal arachnoid adhesion. ${ }^{1,5}$

To understand the pathophysiology of adhesive arachnoid pathology, microanatomy of the spinal subarachnoid space should be considered. Anatomy of the spinal subarachnoid space is different on the dorsal and ventral spinal cord surface.$^{7-10}$ The dorsal subarachnoid space has rich arachnoid trabeculation, and a mesh-like structure is present at the midline (the midline dorsal septum). ${ }^{9,10}$ This midline dorsal septum is wider at the dorsal spinal cord surface and covers the dorsal spinal veins. There is also the dorsolateral septum covering the posterior rootlets on each side. ${ }^{7}$ On the other hand, there are no arachnoid trabeculae in the ventral spinal subarachnoid space. ${ }^{9,10}$ Considering these anatomical structures, the idiopathic form is likely to originate from adhesive change of the midline dorsal septum and bilateral dorsolateral septa. The adhesion results in a web-like membranous structure in the dorsal subarachnoid space. Such membranous structures receive pulsatile CSF flow and produce compressive effects on the dorsal spinal cord. ${ }^{2}$ Adhesions at the multiple levels result in cyst formation. Because the dorsal subarachnoid space is larger and pulsatile CSF movement is more dynamic at the thoracic levels, symptomatic adhesion of the dorsal arachnoid septa may occur more frequently than at other spinal levels.

In hemorrhagic or traumatic disorders involving spinal subarachnoid space, the dorsal subarachnoid space is most prone to adhesive changes because of rich arachnoid trabeculae and veins. These structures capture clots, debris, or inflammatory cells and cause severe adhesive change. The arachnoid membrane is thickened and develops fibrous changes with vascular proliferation. The 

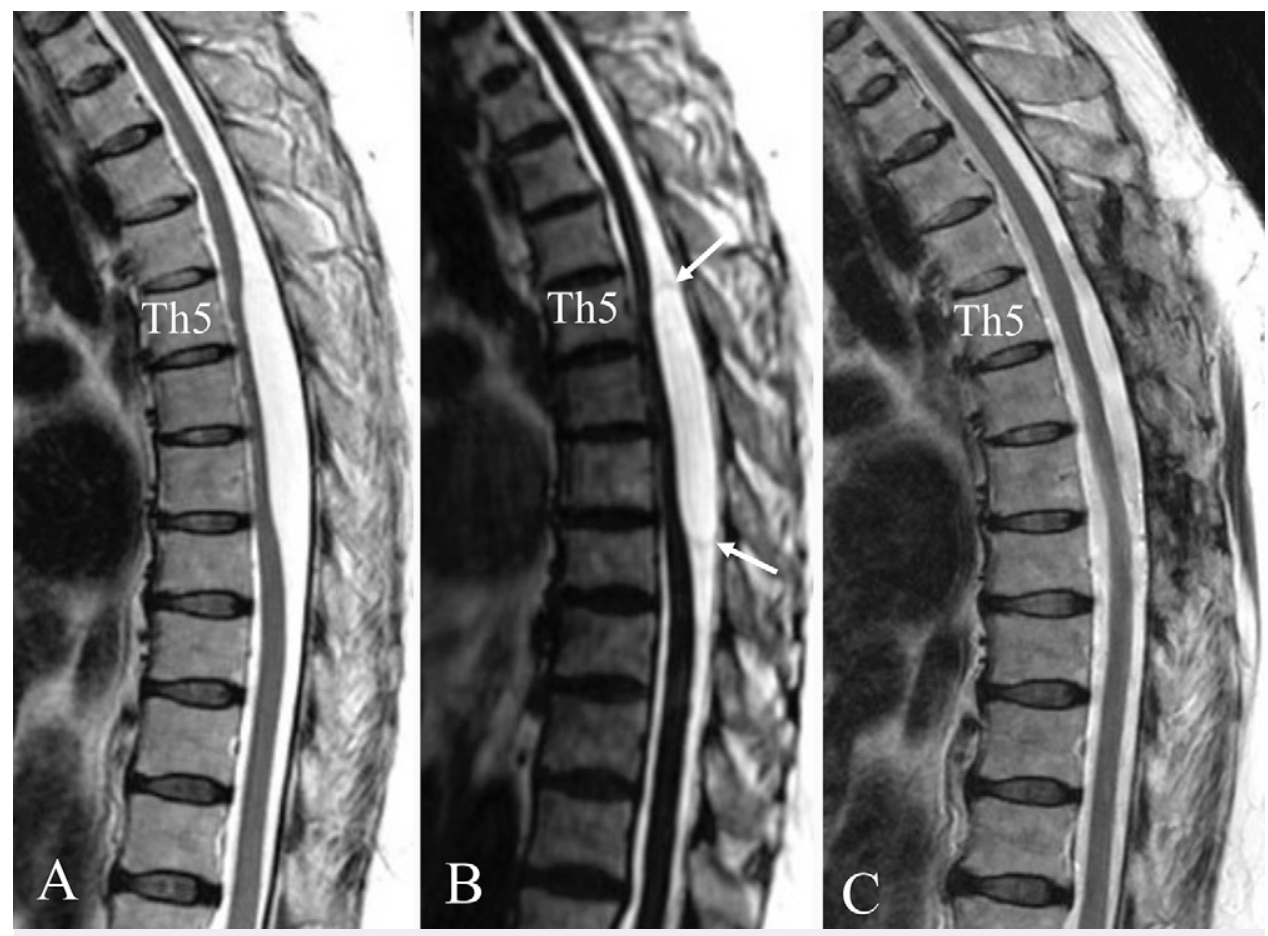

FIG. 4. Pre- and postoperative MRI findings of the thoracic spine of Case 15. This patient presented with thoracic pain and mild paraparesis 3 years before admission without etiological disorders. A: Preoperative T2-weighted sagittal image showed localized dilatation of the dorsal subarachnoid space and displaced spinal cord at the T5-7 levels. B: Heavy T2-weighted sagittal image (FIESTA) demonstrating cyst wall in the dorsal subarachnoid space (arrows). The patient received T5-7 laminectomy and removal of subarachnoid cyst wall. C: Postoperative T2-sagittal image 4 years after surgery showed normalized subarachnoid space. The patient showed good recovery of symptoms.

thickened arachnoid membrane also adheres to the inner surface of the dura, and the proliferated vessels of the arachnoid membrane are communicated with dural vasculatures. This process further facilitates fibrous changes of the arachnoid membrane. The ventral subarachnoid space does not have arachnoid trabeculae and is relatively resistant to adhesive changes. However, diffuse involvement of the spinal subarachnoid space causes both dorsal and ventral adhesions, resulting in disturbed CSF flow and constrictive effects on the veins of the spinal cord. These circulatory disturbances are the main mechanisms of myelopathy in secondary pathology.

In the present study, MRI revealed interstitial edema of the spinal cord in $16(84.2 \%)$ and syrinx formation in $13(68.4 \%)$ of 19 patients. The exact mechanism of intramedullary fluid retention was not elucidated. It is known that a substantial amount of interstitial fluid derives from the arteriolar end of the capillaries and drains into venous channels from the venular end of capillaries in the systemic circulation. The interstitial fluid dynamics are the same in the spinal cord although the spinal cord has other fluid pathways, such as perivascular spaces and the central canal. Because of anatomical structures, the dorsal spinal veins are directly influenced by the subarachnoid CSF pressure and covering arachnoid trabeculae. ${ }^{11}$ Adhesive changes of the dorsal subarachnoid space cause constrictive or compressive effects on the dorsal spinal veins. Indeed, we often observed compressed spinal cord veins under the thickened arachnoid membrane during lysis surgery in patients with the secondary arachnoid pathology (Figs. 1 and 2). Such constrictive dorsal veins decrease absorption capacity of the interstitial fluid from the intramedullary venous tributaries and cause retention of interstitial fluid in the spinal cord. ${ }^{11}$

From our results, surgical treatment of patients with idiopathic pathology was effective because adhesive change was localized for short segments in the dorsal subarachnoid space. However, it should be noted that preoperative diagnosis was often delayed (onset to admission: 4 to 120 months in our series), probably because the main symptom was back or thoracic pain and MRI showed only small cystic dilatation of the dorsal subarachnoid space. Visualization of the cyst wall by heavily T2weighted MRI such as fast imaging using steady-state acquisition (FIESTA) is helpful for correct diagnosis (Figs. 3 and 4).

In contrast, results of surgical treatment of patients with the secondary pathology were not satisfactory. Although lysis of arachnoid adhesion is the primary surgical strategy for this type, $3(27.3 \%)$ of 11 patients who received lysis procedure in our series showed neurological deterioration immediately after surgery, probably because of damage to the spinal cord or pial vessels during subarachnoid lysis. Because disturbed venous circulation of the spinal cord by arachnoid adhesion is one of the important mechanisms of myelopathy, removal of the thickened fibrous part of the arachnoid membrane and preservation of thin arachnoid covering dorsal spinal veins are safe decompressive methods of lysis surgery. Lysis of adhesive arachnoid membrane to the inner surface of the dura may be effective in preventing fibrotic process by interrupting dura-arachnoid vascular connection. 
In our series, S-S shunt was performed in $9(69.2 \%)$ of 13 patients with secondary pathology as the first (5 patients) or second (4 patients) surgical treatment. Interestingly, follow-up MRI revealed that long-term collapse of the syrinx was achieved in 8 of 9 patients. In most of these patients, the S-S shunt was placed at the level of almost normal subarachnoid space. Because the syrinx fluid presumably derives from excessive interstitial fluid in the spinal cord, drainage of fluid into the normal pathway of CSF circulation is a reasonable surgical procedure. From our experience, the effect of an S-S shunt was mainly alleviation of pain or numbness. Although an S-S shunt should not be indicated for the spinal cord with subarachnoid adhesion, this surgical procedure is beneficial for patients showing extended syrinx from the adhesion level.

\section{Lessons}

The present study showed two types of spinal adhesive pathology. Idiopathic pathology was the localized adhesion causing dorsal arachnoid cyst formation predominantly at the thoracic level. Surgical resection of cyst wall was effective, although careful MRI examination was needed for correct diagnosis of cyst wall. The other type was secondary pathology caused by various disorders affecting spinal subarachnoid space. Disturbed venous circulation of the spinal cord due to extensive arachnoid adhesion was the main mechanism of myelopathy. Lysis of arachnoid adhesion was the primary surgical treatment for this type. However, subarachnoid lysis had a risk of postoperative neurological deficits. Careful removal of the thickened membrane with preservation of arachnoid layer covering the spinal cord vessels may provide safe decompressive lysis. If the syrinx extends to the level of normal subarachnoid space, an S$S$ shunt will be effective for long-term collapse of the syrinx. Surgical strategy for the secondary pathology should include S-S shunt in selected patients in addition to lysis surgery.

\section{References}

1. Koyanagi I, Iwasaki Y, Hida K, Houkin K. Clinical features and pathomechanisms of syringomyelia associated with spinal arachnoiditis. Surg Neurol. 2005;63(4):350-356.

2. Baba T, Koyanagi I, Yoshifuji K, Murakami T, Houkin K. Pulsatile wall movement of spinal arachnoid cyst deteriorates spinal cord symptoms: report of three cases. Acta Neurochir (Wien). 2010; 152(7):1245-1249.
3. Perret G, Green D, Keller J. Diagnosis and treatment of intradural arachnoid cysts of the thoracic spine. Radiology. 1962;79:425-429.

4. Nisson PL, Hussain I, Härtl R, Kim S, Baaj AA. Arachnoid web of the spine: a systematic literature review. J Neurosurg Spine. 2019;31(2):1-10.

5. Klekamp J. A new classification for pathologies of spinal meninges. Part 2: Primary and secondary intradural arachnoid cysts. Neurosurgery. 2017;81(2):217-229.

6. McCormick PC, Torres R, Post KD, Stein BM. Intramedullary ependymoma of the spinal cord. J Neurosurg. 1990;72(4):523-532.

7. Nauta HJ, Dolan E, Yasargil MG. Microsurgical anatomy of spinal subarachnoid space. Surg Neurol. 1983;19(5):431-437.

8. Nicholas DS, Weller RO. The fine anatomy of the human spinal meninges. A light and scanning electron microscopy study. J Neurosurg. 1988;69(2):276-282.

9. Tator $\mathrm{CH}$, Koyanagi I. Vascular mechanisms in the pathophysiology of human spinal cord injury. J Neurosurg. 1997;86(3):483-492.

10. Parkinson D. Human spinal arachnoid septa, trabeculae, and "rogue strands." Am J Anat. 1991;192(4):498-509.

11. Koyanagi I, Houkin K. Pathogenesis of syringomyelia associated with Chiari type 1 malformation: review of evidence and proposal of a new hypothesis. Neurosurg Rev. 2010;33(3):271-285.

\section{Disclosures}

The authors report no conflict of interest concerning the materials or methods used in this study or the findings specified in this paper.

\section{Author Contributions}

Conception and design: Koyanagi. Acquisition of data: Koyanagi, Chiba, Yoshino. Analysis and interpretation of data: Koyanagi. Drafting the article: Koyanagi. Critically revising the article: Aida. Approved the final version of the manuscript on behalf of all authors: Koyanagi. Statistical analysis: Koyanagi. Administrative/technical/material support: Uemori, Aida. Study supervision: Imamura, Aida.

\section{Supplemental Information}

Previous Presentations

A part of this study was presented as an e-poster at the 2021 AANS Annual Scientific Meeting, August 21-25, 2021.

\section{Correspondence}

Izumi Koyanagi: Hokkaido Neurosurgical Memorial Hospital, Sapporo, Hokkaido, Japan. koyanagi@hnsmhp.or.jp. 